\title{
APPLICATION OF DIFFERENTIAL TRANSFORM METHOD TO THE HYPER-SINGULAR INTEGRAL EQUATIONS
}

\author{
Mohammad Abdulkawi Mahiub \\ Department of Mathematics \\ Faculty of Science and Arts \\ Najran University, SAUDI ARABIA
}

\begin{abstract}
In this manuscript, the differential transform method is extended to solve the hyper-singular integral equations. Three theorems for transformation of integrals are given with proofs. Approximate solutions of hyper-singular integral equations with two types of kernels. Properties, such degenerate and convolution, are investigated.

Numerical results are shown to illustrate the efficiency and accuracy of the present method.
\end{abstract}

AMS Subject Classification: 65R20, 45E05, 45E10, 35A22

Key Words: hyper singular integral equations, convolution kernel, degenerate kernel, differential transform method

\section{Introduction}

Consider the Hyper-singular integral equations (HSIEs) of the form

$$
f_{-1}^{1} \frac{\psi(t)}{(t-x)^{2}} d t+\int_{-1}^{1} K(x, t) \psi(t) d t=f(x), \quad-1 \leq x \leq 1,
$$

with the conditions

$$
\psi( \pm 1)=0
$$

where $K(x, t)$ is a regular square-integrable function of $t$ and $x, f(x)$ is smooth and $\psi$ is unknown function to be determined. The first integral in Eq.(1) is understood in the sense of Hadmard finite part, that is

$\begin{array}{lrc}\text { Received: } & \text { May 7, } 2016 & \text { (C) } 2017 \text { Academic Publications, Ltd. } \\ \text { Revised: } & \text { January 4, } 2017 & \text { url: www.acadpubl.eu } \\ \text { Published: } & \text { February 1, } 2017 & \end{array}$




$$
f_{-1}^{1} \frac{\psi(t)}{(t-x)^{2}} d t=\lim _{\epsilon \rightarrow 0}\left[\int_{-1}^{x-\epsilon} \frac{\psi(t)}{(t-x)^{2}} d t+\int_{x+\epsilon}^{1} \frac{\psi(t)}{(t-x)^{2}} d t-\frac{2 \psi(x)}{\epsilon}\right] .
$$

Hype-rsingular integral can be defined as the differentiation of Cauchy singular integral

$$
f_{-1}^{1} \frac{\varphi(t)}{(t-x)^{2}} d t=-\frac{d}{d x} \int_{-1}^{1} \frac{\varphi(t)}{t-x} d t
$$

where the integral in the second hand-side is understood in the sense of Cauchy principle-value integral, that is

$$
\int_{-1}^{1} \frac{\psi(t)}{t-x} d t=\lim _{\epsilon \rightarrow 0}\left[\int_{-1}^{x-\epsilon} \frac{\psi(t)}{t-x} d t+\int_{x+\epsilon}^{1} \frac{\psi(t)}{t-x} d t\right]
$$

Eq.(1) arises in a variety of mixed boundary value problems in mathematical physics such as water wave scatting and radiation problems involving thin submerged, fracture mechanics, acoustics, fluid mechanics, and elasticity (see [1], [2], [3]). Chen and Zhou [4] provided a new simple and efficient method for solving HSIEs of the first kind. They used a transform to eliminate the singularity of the equation. Abdulkawi and Al-shameri. [5] extended the differential transform method for solving the characteristic hyper singular integral equation. Mahiub et al. [6] presented an accurate numerical solution for solving HSIE. They used Chebyshev orthogonal polynomials of the second kind for approximating the unknown function. Mandal and Bhattacharya [7] obtained approximate solutions for a simple HSIE and a HSIE of second kind using Bernstein polynomials as basis. Chakrabarti [8] developed a direct function theoretic method to determine the solution of HSIE. Iovane, et al.[9] investigated on direct numerical treatment of HSIE arising in mechanics and acoustics. Mandal and Bera [10] discussed an approximate solution for a class of HSIEs. Dutta and Banerjea [11] solved a HSIE in two disjoint intervals using the solution of Cauchy type singular integral equation in disjoint intervals. They also used a direct function theoretic method to determine the solution of same HSIE in two disjoint intervals. Martin [12] discussed the exact solution of the simplest one-dimensional HSIE and obtained the general solution. Ervin and Stephan [13] analyzed a collocation method for HSIE. They used the Chebyshev polynomials of the second kind as the basis functions for the approximation, and choosed Chebyshev quadrature points as the collocation points.

In this paper, we present a numerical solution for solving the HSIE(1) using Differential transform method (DTM). 


\section{Differential Transform Method}

The Transformation of the $n$th derivative of a function $f$ in one variable is as follows [14]

$$
F(k)=\frac{1}{k !}\left[\frac{d^{k} f(x)}{d x^{k}}\right]_{x=x_{0}}
$$

and the inverse transformation is defined as

$$
f(x)=\sum_{k=0}^{\infty} F(k)\left(x-x_{0}\right)^{k} .
$$

Theorem 1. If $f(x)=x^{n}$, then $F(k)=\delta(k-n)$, where

$$
\delta(k-n)= \begin{cases}1, & k=n, \\ 0, & k \neq n .\end{cases}
$$

Theorem 2. If $f(x)=a g(x)$, then $F(k)=a G(k)$, where $a$ is a constant and $G(k)$ is the differential transform of $g(x)$.

Theorem 3. If $f(x)=g(x) h(x)$, then

$$
F(k)=\sum_{k_{1}=0}^{k} G\left(k_{1}\right) H\left(k-k_{1}\right),
$$

where $G(k)$ and $H(k)$ are the differential transform of $g$ and $h$ respectively.

Theorem 4. If $f(x)=\frac{d^{n} g(x)}{d x^{n}}$, then

$$
F(k)=\frac{(k+n) !}{k !} G(k+n),
$$

where $G(k)$ is the differential transform of $g$.

\section{Numerical Solution of HSIE (1)}

Rewriting the unknown function $\psi(t)$ in Eq.(1) which satisfies the conditions in (2) as

$$
\psi(t)=\sqrt{1-t^{2}} \varphi(t)
$$

where the function $\varphi(t)$ is well behavior on the interval $[-1,1]$. 
Substituting Eq.(8) int Eq.(1) yields

$$
f_{-1}^{1} \frac{\sqrt{1-t^{2}} \varphi(t)}{(t-x)^{2}} d t+\int_{-1}^{1} \sqrt{1-t^{2}} K(x, t) \varphi(t) d t=f(x) .
$$

To obtain the numerical solution of Eq.(9) we need to prove and use the following Theorems.

Theorem 5. If $g_{1}(x)=f_{-1}^{1} \frac{\sqrt{1-t^{2}} \varphi(t)}{(t-x)^{2}} d t$, then the differential transform of $g_{1}$ is

$$
\begin{aligned}
& G_{1}(\bar{k})= \\
& -\sum_{k=1}^{N} \sum_{k_{1}=0}^{k-1} \Phi(k) k_{1}\left[h\left(k-k_{1}-1\right)-h\left(k-k_{1}+1\right)\right] \delta\left(\bar{k}-k_{1}+1\right) \\
& +\pi\left[\Phi(\bar{k})+\sum_{k_{2}=0}^{\bar{k}} \frac{\left(\bar{k}-k_{2}+1\right) !}{\left(\bar{k}-k_{2}\right) !} \Phi\left(\bar{k}-k_{2}+1\right) \delta\left(k_{2}-1\right)\right], N \rightarrow \infty
\end{aligned}
$$

where $\Phi(k)$ is the differential transform of $\varphi$.

Proof. From (4) we have

$$
g_{1}(x)=f_{-1}^{1} \frac{\sqrt{1-t^{2}} \varphi(t)}{(t-x)^{2}} d t=-\frac{d}{d x} \int_{-1}^{1} \frac{\sqrt{1-t^{2}} \varphi(t)}{t-x} d t
$$

Let

$$
g(x)=\int_{-1}^{1} \frac{\sqrt{1-t^{2}} \varphi(t)}{t-x} d t
$$

Rewriting $g(x)$ as

$$
\begin{aligned}
g(x) & =\int_{-1}^{1} \frac{\sqrt{1-t^{2}}[\varphi(t)-\varphi(x)]}{t-x} d t+\varphi(x) \int_{-1}^{1} \frac{\sqrt{1-t^{2}}}{t-x} d t . \\
& =\int_{-1}^{1} \frac{\sqrt{1-t^{2}}[\varphi(t)-\varphi(x)]}{t-x} d t-\pi x \varphi(x) .
\end{aligned}
$$

It is know that

$$
\frac{t^{k}-x^{k}}{t-x}=\sum_{k_{1}=0}^{k-1} x^{k_{1}} t^{k-1-k_{1}}
$$


Using Maclaurin series and relation (13) yields

$$
\begin{aligned}
& \int_{-1}^{1} \frac{\sqrt{1-t^{2}}[\varphi(t)-\varphi(x)]}{t-x} d t \\
& =\sum_{k=1}^{\infty} \Phi(k) \int_{-1}^{1} \frac{\sqrt{1-t^{2}}\left[t^{k}-x^{k}\right]}{t-x} d t \\
& =\sum_{k=1}^{\infty} \Phi(k) \sum_{k_{1}=0}^{k-1} x^{k_{1}} \int_{-1}^{1} \sqrt{1-t^{2}} t^{k-k_{1}-1} d t \\
& =\sum_{k=1}^{\infty} \Phi(k) \sum_{k_{1}=0}^{k-1} x^{k_{1}} \int_{-1}^{1} \frac{t^{k-k_{1}-1}-t^{k-k_{1}+1}}{\sqrt{1-t^{2}}} d t .
\end{aligned}
$$

It is not difficult to verify that

$$
h(m)=\int_{-1}^{1} \frac{t^{m}}{\sqrt{1-t^{2}}} d t= \begin{cases}\pi, & m=0 \\ 0, & m=1,3, \ldots \\ \frac{1 \cdot 3 \cdot 5 \cdots(m-1)}{2^{\frac{m}{2}}\left(\frac{m}{2}\right) !} \pi, & m=2,4, \ldots\end{cases}
$$

From (12), (14) and (15) we obtain

$$
g(x)=\sum_{k=1}^{\infty} \Phi(k) \sum_{k_{1}=0}^{k-1} x^{k 1}\left[h\left(k-k_{1}-1\right)-h\left(k-k_{1}+1\right)\right]-\pi x \varphi(x) .
$$

and then

$$
\begin{aligned}
\frac{d g(x)}{d x}= & \sum_{k=1}^{\infty} \Phi(k) \sum_{k_{1}=0}^{k-1} k_{1} x^{k_{1}-1}\left[h\left(k-k_{1}-1\right)-h\left(k-k_{1}+1\right)\right] \\
& -\pi\left[\varphi(x)+x \frac{d \varphi(x)}{d x}\right] .
\end{aligned}
$$

Thus

$$
\begin{aligned}
g_{1}(x)= & -\sum_{k=1}^{\infty} \Phi(k) \sum_{k_{1}=0}^{k-1} k_{1} x^{k_{1}-1}\left[h\left(k-k_{1}-1\right)-h\left(k-k_{1}+1\right)\right] \\
& +\pi\left[\varphi(x)+x \frac{d \varphi(x)}{d x}\right] .
\end{aligned}
$$


Let $G_{1}(\bar{k})$ be the differential transform of $g_{1}(x)$. Then by using Theorems 1 4 we obtain

$$
\begin{aligned}
& G_{1}(\bar{k})= \\
& -\sum_{k=1}^{\infty} \sum_{k_{1}=0}^{k-1} \Phi(k) k_{1}\left[h\left(k-k_{1}-1\right)-h\left(k-k_{1}+1\right)\right] \delta\left(\bar{k}-k_{1}+1\right) \\
& +\pi\left[\Phi(\bar{k})+\sum_{k_{2}=0}^{\bar{k}} \frac{\left(\bar{k}-k_{2}+1\right) !}{\left(\bar{k}-k_{2}\right) !} \Phi\left(\bar{k}-k_{2}+1\right) \delta\left(k_{2}-1\right)\right]
\end{aligned}
$$

Theorem 6. If $g(x)=\int_{-1}^{1} \sqrt{1-t^{2}} K(x, t) \varphi(t) d t$ where $K(x, t)$ is a degenerate kernel i.e.

$$
K(x, t)=g_{1}(x) g_{2}(t)
$$

then

$$
\begin{aligned}
& G(\bar{k})= \\
& \left(\sum_{k_{1}=0}^{N} \sum_{k_{2}=0}^{N} G_{2}\left(k_{1}\right) \Phi\left(k_{2}\right)\left[h\left(k_{1}+k_{2}\right)-h\left(k_{1}+k_{2}+2\right)\right] G_{1}(\bar{k}),\right. \\
& N \rightarrow \infty
\end{aligned}
$$

where $G_{1}(\bar{k})$ and $G_{2}\left(k_{1}\right)$ are the differential transform of $g_{1}$ and $g_{2}$ respectively.

Proof. Let

$$
\begin{aligned}
g(x) & =\int_{-1}^{1} \sqrt{1-t^{2}} g_{1}(x) g_{2}(t) \varphi(t) d t \\
& =g_{1}(x) \int_{-1}^{1} \sqrt{1-t^{2}} g_{2}(t) \varphi(t) d t .
\end{aligned}
$$

Using Maclaurin series we have

$$
\begin{aligned}
g(x) & =g_{1}(x) \int_{-1}^{1} \sqrt{1-t^{2}}\left(\sum_{k_{1}=0}^{\infty} G_{2}\left(k_{1}\right) t^{k_{1}}\right)\left(\sum_{k_{2}=0}^{\infty} \Phi\left(k_{2}\right) t^{k_{2}}\right) d t \\
& =g_{1}(x) \sum_{k_{1}=0}^{\infty} G_{2}\left(k_{1}\right) \sum_{k_{2}=0}^{\infty} \Phi\left(k_{2}\right) \int_{-1}^{1} \sqrt{1-t^{2}} t^{k_{1}+k_{2}} d t
\end{aligned}
$$




$$
\begin{aligned}
& =g_{1}(x) \sum_{k_{1}=0}^{\infty} G_{2}\left(k_{1}\right) \sum_{k_{2}=0}^{\infty} \Phi\left(k_{2}\right) \int_{-1}^{1} \frac{t^{k_{1}+k_{2}}-t^{k_{1}+k_{2}+2}}{\sqrt{1-t^{2}}} d t \\
& =g_{1}(x) \sum_{k_{1}=0}^{\infty} G_{2}\left(k_{1}\right) \sum_{k_{2}=0}^{\infty} \Phi\left(k_{2}\right)\left[h\left(k_{1}+k_{2}\right)-h\left(k_{1}+k_{2}+2\right)\right] .
\end{aligned}
$$

Using Theorem 2, we obtain

$$
\begin{aligned}
& G(\bar{k})= \\
& \left(\sum_{k_{1}=0}^{\infty} \sum_{k_{2}=0}^{\infty} G_{2}\left(k_{1}\right) \Phi\left(k_{2}\right)\left[h\left(k_{1}+k_{2}\right)-h\left(k_{1}+k_{2}+2\right)\right], G_{1}(\bar{k}) .\right.
\end{aligned}
$$

Theorem 7. If $f(x)=\int_{-1}^{1} \sqrt{1-t^{2}} K(x, t) \varphi(t) d t$, where $K(x, t)$ is a convolution type kernel i.e.

$$
K(x, t)=g(x-t)
$$

then

$$
\begin{aligned}
F(\bar{k})= & \sum_{k_{1}=0}^{N} \sum_{k_{2}=0}^{N} \sum_{k=0}^{k_{2}}(-1)^{k}\left(\begin{array}{c}
k_{2} \\
k
\end{array}\right) \Phi\left(k_{1}\right) G\left(k_{2}\right) \\
& \times\left[h\left(k+k_{1}\right)-h\left(k+k_{1}+2\right)\right] \delta\left(k_{2}-k-\bar{k}\right), \quad N \rightarrow \infty
\end{aligned}
$$

where $G\left(k_{2}\right)$ and $F(\bar{k})$ are the differential transform of $g$ and $F$ respectively.

Let

$$
f(x)=\int_{-1}^{1} \sqrt{1-t^{2}} g(x-t) \varphi(t) d t .
$$

Using Maclaurin series and Binomial series we have

$$
\begin{aligned}
& f(x)=\int_{-1}^{1} \sqrt{1-t^{2}}\left(\sum_{k_{1}}^{\infty} \Phi\left(k_{1}\right) t^{k_{1}}\right)\left(\sum_{k_{2}=0}^{\infty} G\left(k_{2}\right)(x-t)^{k_{2}}\right) d t \\
& =\sum_{k_{1}=0}^{\infty} \sum_{k_{2}=0}^{\infty} \sum_{k=0}^{k_{2}}(-1)^{k}\left(\begin{array}{c}
k_{2} \\
k
\end{array}\right) \Phi\left(k_{1}\right) G\left(k_{2}\right) x^{k_{2}-k} \int_{-1}^{1} \sqrt{1-t^{2}} t^{k+k_{1}} d t \\
& =\sum_{k_{1}=0}^{\infty} \sum_{k_{2}=0}^{\infty} \sum_{k=0}^{k_{2}}(-1)^{k}\left(\begin{array}{c}
k_{2} \\
k
\end{array}\right) \Phi\left(k_{1}\right) G\left(k_{2}\right) x^{k_{2}-k} \int_{-1}^{1} \frac{t^{k+k_{1}}-t^{k+k_{1}+2}}{\sqrt{1-t^{2}}} d t
\end{aligned}
$$




$$
\begin{aligned}
=\sum_{k_{1}=0}^{\infty} \sum_{k_{2}=0}^{\infty} \sum_{k=0}^{k_{2}}(-1)^{k}\left(\begin{array}{c}
k_{2} \\
k
\end{array}\right) \Phi\left(k_{1}\right) G\left(k_{2}\right) x^{k_{2}-k} \\
\times\left[h\left(k+k_{1}\right)-h\left(k+k_{1}+2\right)\right] .
\end{aligned}
$$

Applying Theorem 2 we obtain

$$
\begin{aligned}
F(\bar{k})= & \sum_{k_{1}=0}^{N} \sum_{k_{2}=0}^{N} \sum_{k=0}^{k_{2}}(-1)^{k}\left(\begin{array}{c}
k_{2} \\
k
\end{array}\right) \Phi\left(k_{1}\right) G\left(k_{2}\right) \\
& \times\left[h\left(k+k_{1}\right)-h\left(k+k_{1}+2\right)\right] \delta\left(k_{2}-k-\bar{k}\right) .
\end{aligned}
$$

Now, we will discuss approximate solutions of Eq.(9) for two types of kernel $K(x, t)$.

Type I: Degenerate Kernel. Consider the degenerate kernel

$$
K(x, t)=g_{1}(x) g_{2}(t) .
$$

By using Theorems 5 and 6, Eq.(9) transform to

$$
\begin{aligned}
& -\sum_{k=1}^{N} \sum_{k_{1}=0}^{k-1} \Phi(k) k_{1}\left[h\left(k-k_{1}-1\right)-h\left(k-k_{1}+1\right)\right] \delta\left(\bar{k}-k_{1}+1\right) \\
& +\pi\left[\Phi(\bar{k})+\sum_{k_{2}=0}^{\bar{k}} \frac{\left(\bar{k}-k_{2}+1\right) !}{\left(\bar{k}-k_{2}\right) !} \Phi\left(\bar{k}-k_{2}+1\right) \delta\left(k_{2}-1\right)\right] \\
& +\left(\sum_{k_{1}=0}^{N} \sum_{k_{2}=0}^{N} G_{2}\left(k_{1}\right) \Phi\left(k_{2}\right)\left[h\left(k_{1}+k_{2}\right)-h\left(k_{1}+k_{2}+2\right)\right], G_{1}(\bar{k})\right. \\
& =F(\bar{k}), \quad N \rightarrow \infty .
\end{aligned}
$$

Thus we obtain the following system of linear equations

$$
\begin{aligned}
& -\sum_{k=1}^{N} \sum_{k_{1}=0}^{k-1} \Phi(k) k_{1}\left[h\left(k-k_{1}-1\right)-h\left(k-k_{1}+1\right)\right] \delta\left(\bar{k}-k_{1}+1\right) \\
& +\pi\left[\Phi(\bar{k})+\sum_{k_{2}=0}^{\bar{k}} \frac{\left(\bar{k}-k_{2}+1\right) !}{\left(\bar{k}-k_{2}\right) !} \Phi\left(\bar{k}-k_{2}+1\right) \delta\left(k_{2}-1\right)\right] \\
& +\left(\sum_{k_{1}=0}^{N} \sum_{k_{2}=0}^{N} G_{2}\left(k_{1}\right) \Phi\left(k_{2}\right)\left[h\left(k_{1}+k_{2}\right)-h\left(k_{1}+k_{2}+2\right)\right] G_{1}(\bar{k})\right.
\end{aligned}
$$




$$
=F(\bar{k}), \quad \bar{k}=0,1,2, \cdots, N .
$$

Therefore, the approximate solution of Eq.(9) with degenerate kernel will be of the form

$$
\varphi(x) \approx \sum_{k=0}^{N} \Phi(k) x^{k}
$$

Type II: Convolution type Kernel. Consider the convolution type kernel

$$
K(x, t)=g(x-t) .
$$

By using Theorems 5 and 7, Eq.(9) transform to

$$
\begin{aligned}
& -\sum_{k=1}^{N} \sum_{k_{1}=0}^{k-1} \Phi(k) k_{1}\left[h\left(k-k_{1}-1\right)-h\left(k-k_{1}+1\right)\right] \delta\left(\bar{k}-k_{1}+1\right) \\
& +\pi\left[\Phi(\bar{k})+\sum_{k_{2}=0}^{\bar{k}} \frac{\left(\bar{k}-k_{2}+1\right) !}{\left(\bar{k}-k_{2}\right) !} \Phi\left(\bar{k}-k_{2}+1\right) \delta\left(k_{2}-1\right)\right\rfloor \\
& +\sum_{k_{1}=0}^{N} \sum_{k_{2}=0}^{N} \sum_{k=0}^{k_{2}}(-1)^{k}\left(\begin{array}{c}
k_{2} \\
k
\end{array}\right) \Phi\left(k_{1}\right) G\left(k_{2}\right)\left[h\left(k+k_{1}\right)-h\left(k+k_{1}+2\right)\right] \\
& \times \delta\left(k_{2}-k-\bar{k}\right)=F(\bar{k}) .
\end{aligned}
$$

Thus we obtain the following system of linear equations

$$
\begin{aligned}
& -\sum_{k=1}^{N} \sum_{k_{1}=0}^{k-1} \Phi(k) k_{1}\left[h\left(k-k_{1}-1\right)-h\left(k-k_{1}+1\right)\right] \delta\left(\bar{k}-k_{1}+1\right) \\
& +\pi\left[\Phi(\bar{k})+\sum_{k_{2}=0}^{\bar{k}} \frac{\left(\bar{k}-k_{2}+1\right) !}{\left(\bar{k}-k_{2}\right) !} \Phi\left(\bar{k}-k_{2}+1\right) \delta\left(k_{2}-1\right) \mid\right. \\
& +\sum_{k_{1}=0}^{N} \sum_{k_{2}=0}^{N} \sum_{k=0}^{k_{2}}(-1)^{k}\left(\begin{array}{c}
k_{2} \\
k
\end{array}\right) \Phi\left(k_{1}\right) G\left(k_{2}\right)\left[h\left(k+k_{1}\right)-h\left(k+k_{1}+2\right)\right] \\
& \times \delta\left(k_{2}-k-\bar{k}\right)=F(\bar{k}), \quad \bar{k}=0,1,2, \cdots, N
\end{aligned}
$$

Therefore, the approximate solution of Eq.(9) with convolution type kernel will be of the form

$$
\varphi(x) \approx \sum_{k=0}^{N} \Phi(k) x^{k}
$$




\section{Numerical Results}

Example 1. Let us consider the HSIE

$$
f_{-1}^{1} \frac{\sqrt{1-t^{2}} \varphi(t)}{(t-x)^{2}} d t+\int_{-1}^{1}(t-x) \sqrt{1-t^{2}} \varphi(t) d t=f(x) .
$$

The exact solution of equation (34) when $f(x)=-\frac{\pi}{2}\left(1-6 x^{2}\right)-\frac{\pi}{8} x$ is

$$
\varphi(x)=x^{2} .
$$

It is easy to see that the differential Transform of $f(x)$ is

$$
\left.\begin{array}{ll}
F(0)=-\frac{\pi}{2} & F(1)=-\frac{\pi}{8}, \\
F(2)=3 \pi, & F(k)=0, k=3,4, \cdots .
\end{array}\right\}
$$

In this example $K(x, t)=g(x-t)=(x-t)$, which is convolution type kernel. Then

$$
G(0)=0, \quad G(1)=-1, \quad G(2)=0\} .
$$

Due to (32) for $N=2$ and relations (36)-(37), we obtain the following system of linear equations

$$
\left.\begin{array}{l}
\pi \Phi(0)+\frac{\pi}{8} \Phi(1)-\frac{\pi}{2} \Phi(2)=-\frac{\pi}{2} \\
\frac{\pi}{2} \Phi(0)+2 \pi \Phi(1)-\frac{\pi}{8} \Phi(2)=-\frac{\pi}{8} \\
3 \pi \Phi(2)=3 \pi
\end{array}\right\}
$$

which has the following solution

$$
\Phi(0)=0, \quad \Phi(1)=0, \quad \Phi(2)=1\} .
$$

Thus the numerical solution of Eq.(34) is

$$
\varphi(x)=x^{2} .
$$

which is identical to the exact solution.

Example 2. Consider the following HSIE

$$
f_{-1}^{1} \frac{\sqrt{1-t^{2}} \varphi(t)}{(t-x)^{2}} d t+\int_{-1}^{1} t x \sqrt{1-t^{2}} \varphi(t) d t=f(x) .
$$


The exact solution of equation (41) when $f(x)=8 \pi x^{3}-\frac{15}{8} \pi x+\pi$ is

$$
\varphi(x)=1+2 x^{3}
$$

It is easy to see that the differential Transform of $f(x)$ is

$$
\left.\begin{array}{l}
F(0)=\pi, \quad F(1)=-\frac{15}{8} \pi, \quad F(2)=0 \\
F(3)=8 \pi, \quad F(k)=0, k=4,5, \cdots .
\end{array}\right\}
$$

In this example $K(x, t)=t x$, which is degenerate type kernel. Then

$$
\left.\begin{array}{llll}
G_{1}(0)=0, & G_{1}(1)=1, & G_{1}(2)=0, & G_{1}(3)=0, \\
G_{2}(0)=0, & G_{2}(1)=1, & G_{2}(2)=0, & G_{2}(3)=0 .
\end{array}\right\}
$$

Due to (31) for $N=3$ and relations (43)-(44), we obtain the following system of linear equations

$$
\left.\begin{array}{l}
-\frac{\pi}{2} \Phi(2)+\pi \Phi(0)=\pi \\
\frac{17}{8} \pi \Phi(1)-\frac{15}{16} \pi \Phi(3)=-\frac{15}{8} \pi \\
3 \pi \Phi(2)=0 \\
4 \pi \Phi(3)=8 \pi .
\end{array}\right\}
$$

which has the following solution

$$
\Phi(0)=1, \quad \Phi(1)=0, \quad \Phi(2)=0, \quad \Phi(3)=2\} .
$$

Thus the numerical solution of Eq.(41) is

$$
\varphi(x)=1+2 x^{3} .
$$

which is identical to the exact solution.

Example 3. Consider the following HSIE

$$
f_{-1}^{1} \frac{\sqrt{1-t^{2}} \varphi(t)}{(t-x)^{2}} d t+\int_{-1}^{1} t \cos (x) \sqrt{1-t^{2}}(t) d t=f(x) .
$$

The exact solution of equation (48) when $f(x)=\frac{\pi}{2}+3 \pi x^{2}$ is

$$
\varphi(x)=1+x^{2}
$$


It is easy to see that the differential Transform of $f(x)$ is

$$
\left.\begin{array}{l}
F(0)=\frac{\pi}{2}, \quad F(1)=0, \\
F(2)=3 \pi, \quad F(k)=0, k=3,4, \cdots .
\end{array}\right\}
$$

Note that $K(x, t)=t \cos x$ which is degenerate kernel. Let $g_{1}(x)=\cos x$ and $g_{2}(t)=t$. Then

$$
G_{1}(k)= \begin{cases}\frac{(-1)^{\frac{k}{2}}}{k !}, & k=0,2,4, \cdots, \\ 0, & k=1,3,5, \cdots .\end{cases}
$$

and

$$
\left.G_{2}(0)=0, \quad G_{2}(1)=1, \quad G_{2}(k)=0, \quad k=2,3, \cdots\right\} .
$$

Due to (31) for $N=2$ and relations (50)-(52), we obtain the following system of linear equations

$$
\left.\begin{array}{l}
\pi \Phi(0)+\frac{\pi}{8} \Phi(1)-\frac{\pi}{2} \Phi(2)=\frac{\pi}{2} \\
2 \pi \Phi(1)=0 \\
3 \pi \Phi(2)-\frac{\pi}{8} \Phi(1)=3 \pi
\end{array}\right\}
$$

which has the following solution

$$
\Phi(0)=1, \quad \Phi(1)=0, \quad \Phi(2)=1\} .
$$

Thus the numerical solution of Eq.(48) is

$$
\varphi(x)=1+x^{2} .
$$

which is identical to the exact solution.

\section{Conclusion}

DTM has been extended for solving HSIEs. Two types of kernels, degenerate and convolution, are considered. In the first example, HSIE with convolution type kernel is considered. In the second and third examples, HSIE with degenerate type kernel is considered.. A symbolic calculation software package, Maple 15, is used in the derivations. From the numerical results, it can be seen that the numerical solutions are identical to the exact solutions for all considered examples which show that the DTM is a reliable tool for the solution of HSIEs. The present method reduces the computational difficulties of the other methods and all the calculations can be made simple manipulations. 


\section{Acknowledgments}

This work was supported by the Scientific Research Deanship in Najran University, Kingdom of Saudi Arabia under research project number NU/ESCI/14/043.

\section{References}

[1] Y. S. Chan, A. C. Fannjiang, G. H. Paulino, Integral equations with hypersingular kernels - theory and applications to fracture mechanics, International Journal of Engineering Science, 41 (2003), 683-720.

[2] J. H. De Klerk, Hypersingular integral equations-past,present, future, Nonlinear analysis, 63 (2005), 533-540.

[3] I. K. Lifanov, L. N. Poltavskii, G. M. Vainikko, Hypersingular integral equations and their applications, CRC Press LLC, (2004).

[4] Zhong Chen, Yong Fang Zhou, A New method for solving hypersingular integral equations of the first kind, Applied Mathematics Letters, 24 (2011), 636-641.

[5] Mohammed Abdulkawi Mahiub, WadiaFaid Hassan Al-shameri, Numerical Solution of Hyper singular integral equations by using Differential Transform Method, European Journal of Scientific Research, 132, No.1 (2015), 83-89.

[6] Mohammad Abdulkawi Mahiub, N.M.A. Nik Long, Z.K. Eshkuvatov, Numerical solution of hypersingular integral equations, International Journal of Pure and Applied Mathematics, 69, No.3 (2011), 265-274.

[7] B. N. Mandal, Subhra Bhattacharya, Numerical solution of some classes of integral equation using Bernstein polynomials, Applied Mathemativs and Computation, 190, No. 2 (2007) 1707-1716.

[8] A. Chakrabarti, Solution of a simple hypersingular integral equations, Journal of Integral Equations and Applications, 19, No. 4 (2007), 465-471.

[9] G. Iovane, I, M. Lifanov, M. Sumbatyan, On direct numerical treatment of hypersingular integral equations arising in mechanics and acoustics, Acta Mechanica, 162 (2003), 99110.

[10] B. N. Mandal, G. H. Bera, Approximate solution for a class of hypersingular integral equations, Applied Mathematics Letters, 19 (2006), 1286-1290.

[11] Barnali Dutta, Sudeshna, Solution of a hypersingular equation in two disjoint intervals, Applied Mathematics Letters, 22 (2009), 1281-1285.

[12] P. A. Martin, Exact solution of a simple hypersingular integral equation, journal of integral equations and applications, 4, No. 2 (1992), 197-204.

[13] V. J, Ervin, E. P. Stephan, Collocation with chebyshev polynomials for a hypersingular integral equation on an interval, Journal of computational and applied Mathematics, 43 (1992), 221-229.

[14] Aytac Arikoglu, Ibrahim Ozkol, Solution of differential-difference equations by using differential transform method, Applied Mathematics and Computation, 181 (2006), 153162. 
